Revista Iberoamericana, Vol. LXIX, Núm. 204, Julio-Septiembre 2003, 555-563

\title{
EL MUNDO ECOCÉNTRICO EN \\ SIETE ÁRBOLES CONTRA EL ATARDECER \\ DE PABLO ANTONIO CUADRA
}

POR

\author{
STEVEN White \\ Saint Lawrence University
}

Según Guillermo Yepes Boscán en su introducción a Siete árboles contra el atardecer (1980), Pablo Antonio Cuadra ha conseguido plasmar a través de la ceiba, el jocote, el panamá, el cacao, el mango, el jenísero, y el jícaro, "la totalidad de lo real nicaragüense” que consiste, dice el crítico venezolano, en una fértil unión de "naturaleza, historia, hombre y mito; pasado y presente, pero también un porvenir posible” (Yepes Boscán 36). Este artículo se organiza en torno a la ecocrítica, un término inventado por William Rueckert en 1978 que se define, según Cheryll Glotfelty y Harold Fromm, como "el estudio de la relación entre la literatura y el medio ambiente físico" (Glotfelty y Fromm xviii). El enfoque será literario, claro, pero con un acercamiento centrado en la tierra, destacando así la suma importancia del sitio, del espacio imaginado de los poemas que funcionan como intermedios entre lo humano y lo más-que-humano. El resultado es un libro-mundo de poesía ecocéntrica basada, según Cuadra, en "la precisión lingüística” de la botánica (White, El Pez y la Serpiente 82).

En Siete árboles contra el atardecer, Cuadra demuestra lo que podría considerarse una doble filiación constituida por la topofilia y la biofilia. La primera, según Yi-Fu Tuan, se compone de todos los vínculos humanos afectivos con el medio ambiente material (Tuan 93), y la segunda, en las palabras de Stephen R. Kellert y Edward O. Wilson, consiste en la necesidad de los seres humanos, como parte de la evolución, de relacionarse profunda e íntimamente con la biota viva de la naturaleza con el fin de poder realizarse en términos estéticos, intelectuales, cognitivos e, incluso espirituales (Kellert y Wilson 201). Es decir, los árboles en el libro de Cuadra tienen una realidad científica que corresponde a su especie y un ecosistema complejo de una región cuyas fronteras no respetan necesaria y exclusivamente los parámetros de Nicaragua como país. Los siete dramatis personae denominados formalmente o en los poemas mismos o en las notas que los acompañan tienen un innegable valor simbólico (como es de esperar en el mundo metafórico de la poesía), pero también una realidad y una vitalidad literales, más allá de lo literario. Eriodendron anfractuosum, Spondias purpurea, Sterculia apetala, Theobroma cacao, Mangifera indica, Pithecellobium saman, y Crescentia cujete forman una parte de la rica biodiversidad del lugar que habita el poeta y ayudan a combatir la homogeneidad y la uniformidad sin alternativas de lo que Vandana Shiva ha llamado los "monocultivos de la mente” (Shiva 7). 
¿Quiénes son estos protagonistas y por qué los escogió el poeta? El marco dramático definido por la referencia a la obra Los siete contra Tebas de Esquilo determina, lógicamente, el número de actores. Cuadra, al utilizar esta obra trágica de la antigüedad clásica como herramienta analógica para crear un contexto macrocósmico, establece una relación compleja entre la flora centroamericana y la defensa de la ciudad de Tebas. Los poemas de Siete árboles contra el atardecer son escritos entre 1977-1978, años que corresponden a la insurrección contra la dictadura de Somoza, un conflicto fratricida que encuentra su reflejo en la guerra entre los dos hermanos Eteocles y Polyneices. Hay una similitud principal entre Siete árboles contra el atardecer y Los siete contra Tebas. Tanto en la obra de Cuadra como la de Esquilo existe una enorme preocupación topofílica. Esquilo establece la importancia del ciclo natural que provee a la vida la estabilidad, uniendo así a sus habitantes por medio de vínculos afectivos a un lugar específico, algo que hace Cuadra a la perfección en este libro de poemas (Thalmann 43).

Los árboles de esta obra, según lo explica el poeta en un prólogo, “ofrecen en sus maderas un bálsamo restañador de heridas, o reavivan, con su follaje y sus frutos, la memoria de la misión libertaria del hombre y su dignidad inclaudicable" (Cuadra, Siete árboles... 10). Estas siete especies de árboles se convierten en hitos de una sensibilidad espacial concebida como un lugar específo cargado de mito, historia y memoria personal. Cuadra intenta hacer visible en el mapa de sus poemas estos elementos de un paisaje invisible. Según Kent C. Ryden, "el sentido de un lugar es el resultado gradual e inconsciente de habitar un paisaje por mucho tiempo, familiarizándose con sus propiedades físicas, acumulando una historia dentro de sus parámetros” (Ryden 38).

En los poemas de Siete árboles contra el atardecer, hay un yo disuelto en el medio ambiente que considera que el escribir poesía es un acto ecológico, como dice en "El Jocote”: “Escucha, pues este poema, sembrador de árboles” (Cuadra, Siete árboles... 47). Lo que hace Cuadra en estos poemas es crear un paisaje por medio de lo vivido con la precisión botánica objetiva y sus propias experiencias subjetivas. En una entrevista que le hice al poeta en enero de 2000, fecha en que celebraba el aniversario de su matrimonio con Adilia Bendaña Ramírez en 1935, Cuadra dice: "Lo que quiero decir es que cada árbol tuvo su razón de ser en mi vida. El jocote, por ejemplo, es una especie de alcahuete, porque fue ahí al pie del árbol donde besé a mi mujer por primera vez. (Lo digo en el poema). Hay una leyenda antigua, una metáfora sensual en torno a ese árbol que bota sus hojas para dar el fruto como la mujer se desnuda para darse: Es el árbol del amor” (White 81). Esta realidad científica y biográfica le da una nueva connotación literal a las relaciones afectivas entre el ser humano y la tierra contenidas en la idea de la topofilia. "El Jocote" describe una correspondencia entre el amor humano y un ciclo natural en que figura hasta un verbo inventado por el pueblo a raíz de las características de este árbol:

Por eso el amor nace en esta tierra cuando los jocotes dan su fruto y los muchachos y las muchachas van a jocotear a los patios y a las huertas y es bajo los árboles que se aman.

(Cuadra, Siete árboles... 45) 
El poeta nos informa que la palabra jocote tiene su origen etimológico en xocotl que en la lengua Nahuatl significa “fruta”. Dice Cuadra además que es



Lo que hay en este fragmento es el rescate imprescindible, a través del conocimiento popular, de una rica biodiversidad que, evidentemente, hay que aprender a cuidar y proteger, ya que la riqueza natural, cultural y lingüística dependen de esta pluralidad. Como aseveran Gary Paul Nabhan y Sara St. Antoine en un aporte sobre la extinción de la experiencia, "No cabe la menor duda que la diversidad lingüística y sus reservas asociadas de conocimiento científico popular han sido más amenazadas en el siglo xx que la misma diversidad biólogica” (Kellert y Wilson 243).

El panamá será siempre el enorme árbol capaz de resistir vientos y huracanes que tenía Cuadra al lado de su casa en la orilla del Gran Lago de Granada. Además de recordarle su amistad con la crítica panameña Gloria Guardia, el árbol, que bajo su “inmensa lámpara verde da luz a la asociación y a la simplicidad”, proporciona al poeta una manera de invocar la vida de los pescadores de esa zona biótica y la (otra vez) impresionante biodiversidad del lago. No hay en este poema ningún esfuerzo por idealizar el paisaje, por crear un locus amoenus abstracto en el sentido renacentista europeo que carece de coordenadas temporales y espaciales. De hecho, en todos los poemas de Siete árboles contra el atardecer el lector se encuentra en la presencia de un medio ambiente con rasgos físicos reales y siempre sabe el dónde y el cuándo de su vivencia del mundo ecocéntrico que construye el poeta.

Nuestra convivencia con la naturaleza, siendo los humanos parte de ella, implica una serie de relaciones agro-económicas y políticas, un fenómeno que se manifiesta con cualidades parecidas a lo largo de la historia humana: el trabajo intensivo de monocultivos apreciados por su valor remunerativo muy alto y un sistema político basado en la militarización para proteger estos intereses económicos. Según Cuadra “El Cacao” señala el paralelo que existe entre el antiguo mundo indígena de los Nahuatl y el mundo contemporáneo globalizado y dominado por la moneda de los Estados Unidos.

La lectura interdisciplinaria de libros como Siete árboles contra el atardecer podría servir para iniciar una nueva especie de alfabetización ecológica que incluye, por supuesto, un conocimiento científico básico sobre la propagación diversa del reino vegetal. El caso del mango es un ejemplo de lo que los científicos llaman adaptive 
radiation, o sea, la diseminación de una especie a diferentes nichos ecológicos, un proceso fomentado por el viento, el agua, los pájaros, los animales y también, claro está, por los seres humanos. El mango para Cuadra pertenece a toda una serie de árboles en movimiento a lo largo de la historia (humana y no-humana) que de alguna manera refleja las peregrinaciones de los habitantes de América en el sentido continental. En un diálogo consigo mismo en "El Mango"el poeta dice:

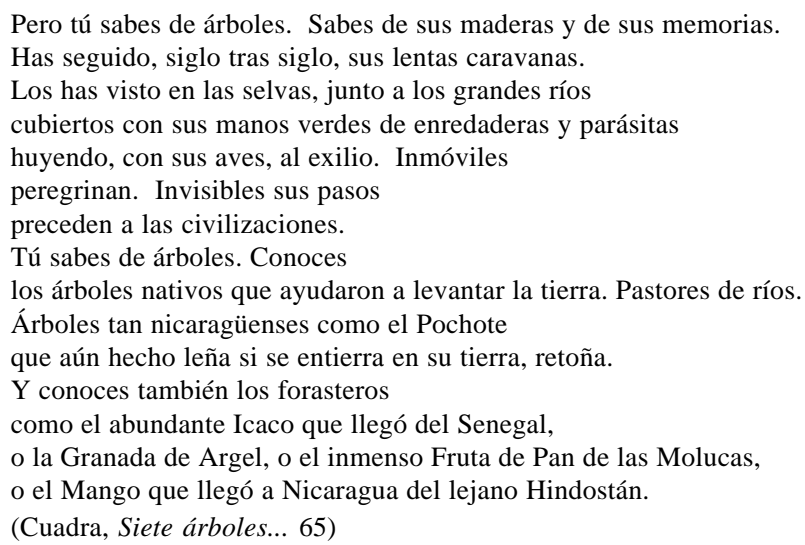

En su estudio fundamental The Environmental Imagination: Thoreau, Nature Writing and the Formation of American Culture, Lawrence Buell habla de la importancia del lector que sabe preguntarse: ¿Cómo actúan los textos como cargadores o agentes de la ecocentricidad? ¿Logra el autor renunciar el mito de la separación humana de la naturaleza? (Buell 143). En Siete árboles contra el atardecer, cada personaje-árbolpoema demuestra cómo la cultura humana está conectada al mundo físico, afectándolo, siendo a la vez, afectada por él. Esta antología vegetal, una verdadera comunidad imaginada de Cuadra, abre un diálogo con sus miembros presentes y además otro con las especies no-incluídas, pero que habitan también el mismo ecosistema. Conny Palacios ha hecho un estudio notable de la obra de Cuadra a través de la perspectiva de una pluralidad de máscaras por medio de las cuales el poeta asume la voz de diferentes personajes que incluyen, entre muchos otros, el chamán, el historiador y el botánico (Palacios 113-30). Siguiendo la idea que expresa A. David Napier en Masks, Transformation and Paradox en cuanto a las máscaras Barong de Bali, yo diría que Cuadra fabrica sus caretas sagradas de la misma madera de las especies que actúan en Siete árboles contra el atardecer (Napier 210-2). Al cubrirse, transformarse, y nutrirse de estos árboles, el asombroso yo del poeta se multiplica en estos poemas, uniéndose con el poder fundador de la naturaleza, sobre todo en "El Jenísero" cuando Cuadra dice:

El rayo: dibujo eléctrico del gran árbol del cosmos. Cierras los ojos al deslumbre y al abrirlos ha nacido el Jenísero. 
Este es el trono de la tormenta.

Pero he aquí que yo he extendido mis ramas y he fundado un reino pacífico. (Cuadra, Siete árboles... 73)

Si "La Ceiba” ayuda a crear la arquitectura materna del cosmos, "El Jenísero” forma su contraparte paterna, describiendo cómo "en la cátedra de este árbol se sienta el sol a distribuir justicia” (Cuadra, Siete árboles... 73). Tanto en este poema como en los otros de Siete árboles contra el atardecer, los árboles son múltiples centros de conocimiento cuyo razonamiento vertical conduce al pensamiento horizontal.

Mientras el jenísero crea un centro, un círculo de verdura que excluye "lo ciego, lo siniestro, lo tenebroso" (Cuadra, Siete árboles... 78), el jícaro se transforma en otro foco de vitalidad redonda que es el ombligo. Como destaca Gutierre Tibón en su fascinante estudio intercultural El ombligo, como centro cósmico: una contribución a la historia de las religiones, "xícalli es la jícara, receptáculo de ombligo por su redondez y largo pedúnculo” (Tibón 51). En cuanto a la estructura geográfica de Tenochtitlán, dice Gutierre que "el centro de la ciudad debía ser el templo primitivo, construído sobre el xicco, la piedra ombligo de Me-Xicco", la ubicación central precisa de la cultura del sacrificio humano del imperio de los Aztecas (Gutierre 187). Según Cuadra en El Nicaragüense, esta idea también corresponde a la región geográfica sincrética que hoy se conoce como Nicaragua: "Hemos sido colocados en un centro mediterráneo: en el ombligo del nuevo mundo” (Cuadra, El nicaragüense 16). El sentido etimológico de la palabra jícaro juega un papel importante en el poema sobre este árbol que cierra Siete árboles contra el atardecer, ya que, en las palabras de Cuadra, "El Jícaro canta la muerte de Pedro Joaquín Chamorro asesinado durante la dictadura de los Somoza” (White 82). Tal como en los otros poemas, el poeta utiliza en "El Jícaro” el lenguaje preciso de la botánica, pero esta vez intentando establecer una relación entre los rasgos físicos del árbol (con sus frutos “como cabezas de hombre”) y su actuación como personaje dramático en el poema:

Sobre este árbol escribo:

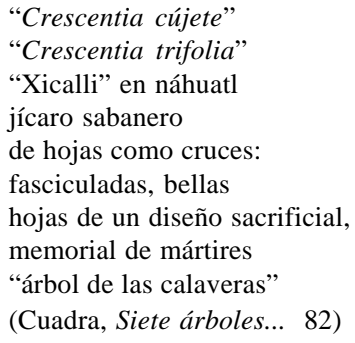

El jícaro, entonces, forma parte de un paisaje que habla, participa en la fundación de una cultura libertaria, dejando su testimonio en un poema del siglo xx (a través del Popol $v u h)$.

Kent C. Ryden habla de distintos niveles de significación que se sobreimponen en el mundo físico. El proceso se realiza por medio de los mitos y el conocimiento folclórico 
que transmiten la historia invisible de un lugar y crean un fuerte sentido de identidad individual y comunitaria (Ryden 62-6). En el caso de este poema de Cuadra, el autor utiliza el jícaro, árbol mítico de la antigua cultura Maya, sí, pero también emblema vivo y presente en el paisaje de Nicaragua, para facilitar la transformación de Chamorro como figura histórica a héroe mítico que ayuda a forjar la libertad de su pueblo.

Uno de los aspectos más importantes de Siete árboles contra el atardecer es cómo establece su "ecopoética". Según Jonathan Bate, se puede entender este término etimológicamente como "un hacer de la morada", ya que la palabra viene del griego poeisis, hacer, y oikos, la casa o el lugar de vivir (Bate 75). Al construir el sitio querido que habita, Cuadra no ignora en sus poemas el amplio valor etnobotánico terapeútico de las especies de árboles que describe en su libro. Cuadra incluye en poemas como "El Jocote”, "El Panamá" (que en la lengua Náhuatl significa "farmacia” o "venta de medicinas”), y "El Cacao" muchas referencias a los usos medicinales de estas especies, basándose en fuentes históricas. Un libro como Siete árboles contra el atardecer perfectamente puede servir para abrir un diálogo sobre dos temas contemporáneos sumamente polémicos e importantes: las plantas transgénicas y los derechos de propiedad intelectual, un fenómeno que suscita (según Paul E. Minnis) preguntas como, ¿Quién es el dueño del conocimiento etnobotánico? y ¿Cómo deben beneficiarse económicamente los pueblos indígenas de la venta de sus tradiciones culturales? (Minnis 5). Últimamente han surgido muchos intentos a nivel internacional de crear un marco legal para resolver este asunto ético, político y económico.

Lo que construye Pablo Antonio Cuadra en Siete árboles contra el atardecer es todo un mundo ecocéntrico. Nos ofrece la oportunidad de considerar la base biofísica de su respuesta estética a una parte determinada del paisaje nicaragüense, es decir, siete especies de árboles. Debido a su manera de existir almacenando la experiencia, estos árbolespoemas conservan en su lugar literalmente el suelo y la memoria locales. Al hablar sobre la pérdida de la narrativa de la flora y fauna (the loss of floral and faunal story), Gary Paul Nabhan y Sara St. Antoine afirman lo siguiente: "Hasta que las tradiciones científicas indígenas trasmitidas oralmente no sean tratadas con el mismo respeto que la ciencia occidental, la monoculture (la palabra “culture” entendida como cultura y también como cultivo) euro-americana continuará extinguiendo una diversidad de respuestas humanas adaptivas al medio ambiente local y, como consecuencia, puede que la biota sea afectada de una manera negativa también” (Nabhan y St. Antoine 248). Mientras tanto, ¿en qué quedamos en cuanto a la esperanza? Jonathan Bate propone que la poesía, en términos muy literales, es la canción de la tierra y se pregunta si el poeta no es una sub-especie clave (keystone sub-species) de Homo sapiens, o sea, el poeta, como una criatura aparentemente inútil pero que es potencialmente el salvador de ecosistemas (Bate 231). En este sentido, los poemas-árboles de Cuadra son canciones de la sustentabilidad. Representan también una técnica mnemónica para recordar quiénes somos, de dónde venimos y, sobre todo, a dónde vamos si no logramos aprender a enraizar el lenguaje en la tierra y cuidar lo que nos sostiene. 
BibliografíA

Abram, David. The Spell of the Sensuous: Perception and Language in a More-ThanHuman World. New York: Vintage, 1997.

Aeschylus. Seven Against Thebes. Anthony Hecht y Helen H. Bacon, trad. New York y London: Oxford University Press, 1973.

Alarcón, Francisco X. Snake Poems: An Aztec Invocation. San Francisco: Chronicle Books, 1992.

Bate, Jonathan. The Song of the Earth. Cambridge: Harvard University Press, 2000.

Buell, Lawrence. The Environmental Imagination: Thoreau, Nature Writing and the Formation of American Culture. Cambridge: Harvard University Press, 1995. Writing for an Endangered World: Literature, Culture and Environment in the U.S. and Beyond. Cambridge: Harvard University Press, 2001.

Caillaux Zazzali, Jorge y Manuel Ruiz Müller, eds. Acceso a recursos genéticos: propuestas e instrumentos jurídicos. Lima: Sociedad Peruana de Derecho Ambiental, 1998.

Cuadra, Pablo Antonio. Siete árboles contra el atardecer y otros poemas. San José, Costa Rica: Libro Libre, 1987.

El Nicragüense. Madrid: Ediciones Cultura Hispánica, 1969.

Elder, John. Imagining the Earth: Poetry and the Vision of Nature. Urbana: University of Illinois Press, 1985.

Eliade, Mircea. The Myth of the Eternal Return Or, Cosmos and History. Princeton: Princeton University Press, 1971.

Esquilo. Tragedias: los siete contra Tebas. Las suplicantes. Madrid: Consejo Superior de Investigaciones Científicas, 1999.

Garibay K., Ángel María. Historia de la Literatura Náhuatl. México: Editorial Porrúa, 1992.

Gifford Terry. Green Voices: Understanding Contemporary Nature Poetry. Manchester: Manchester University Press, 1995.

Glotfelty, Cheryll y Harold Fromm (Eds.). The Ecocriticism Reader: Landmarks in Literary Ecology. Athens/London: University of Georgia Press, 1996.

Greenblatt, Stephen. Marvelous Possessions: The Wonders of the New World. Chicago: University of Chicago Press, 1991.

Hill, Jonathan D. (Ed.) Rethinking History and Myth: Indigenous South American Perspectives on the Past. Urbana/Chicago: University of Illinois Press, 1988.

(Ed.) History, Power, and Identity: Ethnogenesis in the Americas, 1492-1992. Iowa City, University of Iowa Press, 1996.

Johns, Timothy. "Foods and Medicines: Introduction”. Ethnobotany: A Reader. Paul E. Minnis, ed. Norman: University of Oklahoma Press, 2000. 143-7.

Kellert, Stephen R. y Edward O. Wilson (Eds.). The Biophilia Hypothesis. Washington, DC: Island Press, 1993.

Kerridge, Richard and Neil Sammells (Eds.). Writing the Environment: Ecocriticism and Literature. London: Zed, 1998. 
Leon-Portilla, Miguel. Religión de los nicaraos: análisis y comparación de tradiciones culturales nahuas. México: UNAM, 1972.

Mendieta, Rosa María y Silvia del Amo. Plantas medicinales del estado de Yucatán. México: UCECSA, 1981.

Merchant, Carolyn. Earthcare: Women and the Environment. New York: Routledge, 1995.

Minnis, Paul E. (Ed.). Ethnobotany: A Reader. Norman: University of Oklahoma Press, 2000.

Murphy, Patrick D. Farther Afield in the Study of Nature-Oriented Literature. Charlottesville: University Press of Virginia, 2000.

Murray, David. Forked Tongues: Speech, Writing and Representation in North American Indian Texts. Bloomington/Indianapolis: Indiana University Press, 1991.

Napier, A. David. Masks, Transformations, and Paradox. Berkeley: University of California Press, 1986.

Orr, David W. Ecological Literacy: Education and the Transition to a Postmodern World. Albany: State University of New York Press, 1992.

Ortiz de Montellano, Bernard R. Aztec Medicine, Health and Nutrition. New Brunswick: Rutgers University Press, 1990.

Palacios, Conny. Pluralidad de máscaras en la lírica de Pablo Antonio Cuadra. Managua: Academica Nicaragüense de la Lengua, 1996.

Pepper, David. Modern Environmentalism: An Introduction. London: Routledge, 1996.

Rabasa, José. Inventing America: Spanish Historiagraphy and the Formation of Eurocentrism. Norman: University of Oklahoma Press, 1993.

Recinos, Adrián (Ed. y trand.). El Popol vuh: las antiguas historias del Quiché. San José, Costa Rica: EDUCA, 1979.

Ryden, Kent C. Mapping the Invisible Landscape: Folklore, Writing and the Sense of Place. Iowa City: University of Iowa Press, 1993.

Sahagún, Fr. Bernardino de (y los informantes indígenas). El México antiguo. Caracas: Biblioteca Ayacucho, 1981.

Satz, Mario. Arca de roca: ensayos para una sensibilidad ecológica. Barcelona: Editorial Kairós, 1992.

Scigaj, Leonard M. Sustainable Poetry: Four American Ecopoets. Lexington: University Press of Kentucky, 1999.

Shiva, Vandana. Monocultures of the Mind: Perspectives on Biodiversity and Biotechnology. London: Zed, 1993.

Soper, Kate. What Is Nature? Culture, Politics and the Non-Human. Oxford/Cambridge: Blackwell, 1995.

Takacs, David. The Idea of Biodiversity: Philosophies of Paradise. Baltimore: Johns Hopkins University Press, 1996.

Tedlock, Dennis (Ed. y trad.). Popol Vuh. New York: Simon \& Schuster, 1996.

Thalmann, William G. Dramatic Art in Aeschylus's Seven Against Thebes. New Haven/ London: Yale University Press, 1978.

Tibon, Gutierre. El ombligo como centro cósmico: una contibución a la historia de las religiones. México: Fondo de Cultura Económica, 1981. 
Tuan, Yi-Fu. Topophilia: A Study of Environmental Perception, Attitudes and Values. Englewood Cliffs, NJ: Prentice-Hall, 1974.

White, Steven F. “Entrevista a Pablo Antonio Cuadra” (1982). Pablo Antonio Cuadra: valoración múltiple. Jorge Eduardo Arellano, ed. Managua: UNICA, 1994. 95-112. "Entrevista con Pablo Antonio Cuadra”. El Pez y la Serpiente 35 (mayo-junio 2000): 69-84.

Wilson, Edward O. The Diversity of Life. Cambridge: Harvard University Press, 1992.

Yepes Boscán, Guillermo. "Introducción”. Siete árboles contra el atardecer. Pablo Antonio Cuadra. San José, Costa Rica: Libro Libre, 1987.

Zimmerman, Michael E. Contesting Earth’s Future: Radical Ecology and Postmodernity. Berkeley: University of California Press, 1994. 